\title{
Erratum: Understanding bias in the medical literature: With reflections on metastasectomy
}

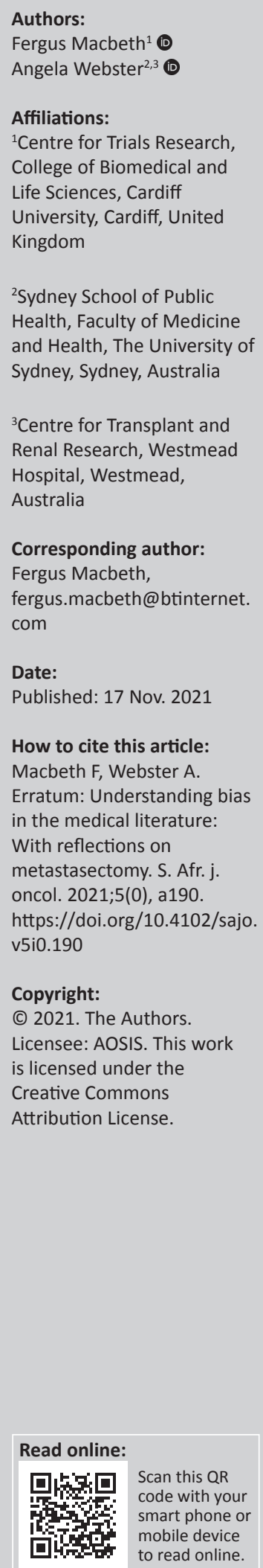
incorrectly.

The sentence is therefore corrected to: journal, Trials. ${ }^{11}$ medical literature: With reflections on metastasectomy. S. Afr. j. oncol. 2020;4(0), a144. https:// doi.org/10.4102/sajo.v4i0.144, on page 4, the journal name, Trails, was misinterpreted and set

Publication bias is seen by the fact that both the CLOCC and SABR-COMET trials were published in relatively high impact oncology journals (JNCI and Lancet, respectively), whereas PulMiCC was turned down by several journals before being published initially in a lower profile, more general open access

This correction does not alter the study's findings of significance or overall interpretation of the study results. The publisher apologises for any inconvenience caused. 